\title{
Bifunctional superlens for simultaneous flexural and acoustic wave
}

\section{superfocusing}

\author{
Yifan Zhu ${ }^{* \dagger}$, Liyun $\mathrm{Cao}^{\dagger}$, Aurélien Merkel, Shi-Wang Fan and Badreddine Assouar ${ }^{*}$ \\ Institut Jean Lamour, CNRS, Université de Lorraine, Nancy, 54000, France.
}

\begin{abstract}
Superfocusing of acoustic and elastic waves is generally achieved by the combination of negative refraction and the enhancement of the evanescent waves. Here, we numerically and experimentally demonstrate the bifunctionality of a superlens that is able to simultaneously focus acoustic and flexural waves beyond the diffraction limit. The designed structure is composed of a two-dimensional arrangement of pillars that act as rigid scatterers for the sound waves and as resonant scatterers for the flexural waves. The band structure presents modes with negative dispersion bands allowing negative refraction for both types of waves within the frequency range 6.9-7.4kHz, which is induced by Bragg scattering effect. Edge modes that enhance the evanescent waves through resonant coupling appear around $7.2 \mathrm{kHz}$ for the flexural and sound wave. The simultaneous superlensing is then observed at this frequency. Our finding will enlighten multi-physical and multi-functional wave manipulations, and could have pragmatic applications involving multi-wave devices.
\end{abstract}

Keywords: Superlensing, Flexural waves, Acoustic waves, Phononic crystal, Multi-functionality.

\section{${ }^{\dagger}$ Equal contribution}

"Corresponding authors: yifan.zhu@univ-lorraine.fr and badreddine.assouar@univ-lorraine.fr 
The concept of superlensing can be traced back to the Pendry's perfect lens in optics [1], which requires both abilities of the convergence of propagating waves and of the participation of non-propagating evanescent waves in the focal spot. The convergence of the waves emerges from the negative refraction of the waves and the evanescent waves are enhanced by surface resonant states that exist at the interface between the lens and the propagating medium. By analogy with the optical devices, acoustic superlensing and superfocusing have been a subject of intense research in the past decade. Indeed, acoustic superlens has been demonstrated using phononic crystals [2-6], acoustic metamaterials [7-10] and acoustic metasurfaces [11-12] for bulk waves, as well as for surface and spoof surface acoustic waves [13-14]. These findings have significant potential applications for wave imaging, due to the fact that super-resolutions are highly desired in imaging system and focused ultrasound is widely used in medical diagnosis.

Although the superlens for one kind of wave has been well demonstrated [1-14], the multiphysics manipulations [15-17] are much less explored and remains a challenging issue to be investigated and elucidated. Here, we propose a concept for a bifunctional superlens with simultaneous controls of flexural and acoustic waves. This concept, illustrated in Fig. 1(a), is based on a two-dimensional (2D) pillar-based phononic crystal attached to a solid plate. The host solid plate can only support $A_{0}$ mode Lamb wave, viz., flexural wave. The incident wave and the output wave are flexural waves in the solid plate. The air medium on the top of the plate can support acoustic wave incidence and transmission. The bifunctionality derives from the design of the phononic crystal, where the pillars can act as rigid scatterers for the airborne waves and, at the same time, as resonant scatterers for the elastic waves. When flexural waves propagate into the phononic crystal, high-order guided elastic mode with negative refractive index will be excited. For acoustic waves, the acoustic impedance of the pillars is much larger than the 
acoustic impedance for air, so that scatterers are regarded as rigid ones. The obtained negative phase velocity mode is related to Bragg scattering effects due to the rigid feature of the scatterers.

As a result, our design is able to simultaneously achieve superlensing beyond the diffraction limit for these two kinds of waves. The multiwave focusing feature of the proposed phononic crystal will have promising applications in energy harvesting, since the multiwave focusing can improve the efficiency of energy harvesting at the elasto-acoustic interface. Our proposed strategy will also enlighten the design of multi-physics and multi-waves device. For example, in previous 2D acoustic wave experiments with parallel waveguide systems [18-19], we can predict that acoustic wave distributions in the air may also influence the elastic wave distributions in the solid waveguide plate. Therefore, it will be interesting to study the elastic (acoustic) wave phenomenon accompanied by acoustic (elastic) wave phenomenon. In addition to the bifunctionality feature, it is worth mentioning that this work has explored the experimental demonstration of flexural wave superlensing. Recently, wave manipulations in thin plates have been actively studied. Indeed, the design of flexural wave lens [20], flexural wave metasurfaces for wavefront manipulations [21-24], and the demonstration of negative refraction [25] or imaging [26] for Lamb waves have been demonstrated. Elastic waves in thin plates have more complex dispersion relation and mode conversion phenomenon in comparison to acoustic waves. Thus, the realization of flexural wave superlens will be of significant interest for wave physics, and have various practical applications, such as nondestructive testing or subwavelength imaging in the solids.

In this research, we consider a thin aluminum plate on which a $2 \mathrm{D}$ phononic crystal will be arranged. The flexural waves propagate in this aluminum plate, having a Young's modulus of 
$E_{a}=70 \times 10^{9} \mathrm{~Pa}$, a mass density of $\rho_{a l}=2700 \mathrm{~kg} / \mathrm{m}^{3}$ and a Poisson's ratio of $v_{a}=0.33$. The plate thickness is of $t_{0}=0.2 \mathrm{~cm}$ on which is glued our phononic crystal as depicted in Figs. 1(a-b). On the other hand, the acoustic waves propagate in the air with a mass density of $\rho_{0}=1.21 \mathrm{~kg} / \mathrm{m}^{3}$ and sound velocity $c_{0}=343 \mathrm{~m} / \mathrm{s}$. The phononic crystal marked by the blue color in Fig. 1(a) is 3Dprinted from poly-lactic acid (PLA) having a Young's modulus of $E_{P L A}=3.2 \times 10^{9} \mathrm{~Pa}$, a mass density of $\rho_{P L A}=1250 \mathrm{~kg} / \mathrm{m}^{3}$, and Poisson's ratio of $v_{P L A}=0.35$ [27-28]. The unit cell of the phononic crystal is shown in Fig. 1(c), corresponding to the red region marked in Fig. 1(a). It consists of two pillars, which are built on a PLA substrate having a thickness $t_{1}=0.2 \mathrm{~cm}$. The lattice constant is $p=4.75 \mathrm{~cm}$. The pillars are composed of two superposed cylinders. The based cylinders of the Pillars 0 and 1 have a radius of $R_{0}=0.96 \mathrm{~cm}$ and $R_{1}=1.44 \mathrm{~cm}$ with a height of $H_{0}=H_{1}=0.5 \mathrm{~cm}$, respectively. The top cylinders have radius of $r_{0}=0.8 \mathrm{~cm}$ and $r_{1}=1 \mathrm{~cm}$ with a height $h_{0}=h_{1}=0.5 \mathrm{~cm}$, respectively. We properly choose the different dimensions of the Pillars 0 and 1 in order to achieve negative refraction in the desired frequency range. The optimization of the structural parameters, viz., the different radius of two stages pillars, help to locate the surface mode within the negative refraction bands, which eventually lead to the superlensing effect for flexural and acoustic waves. 
(a)

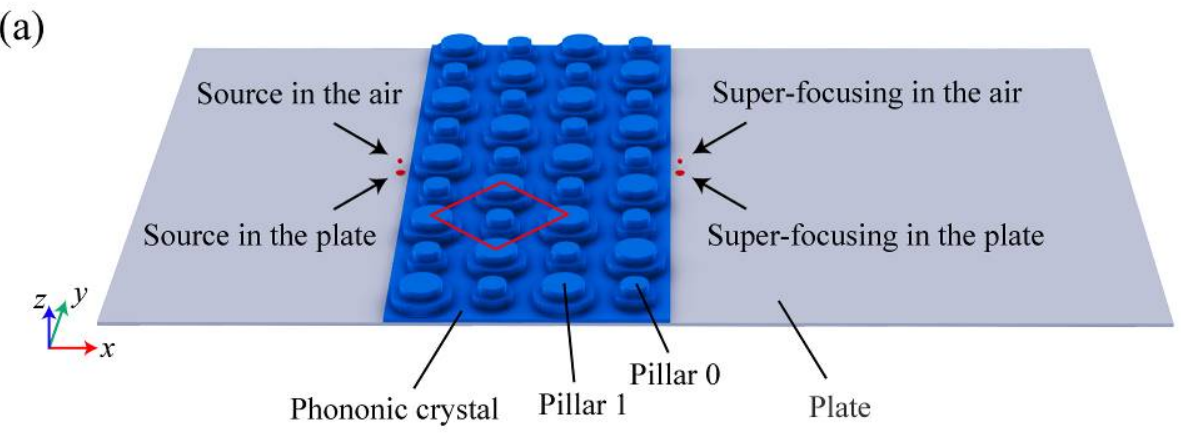

(b)

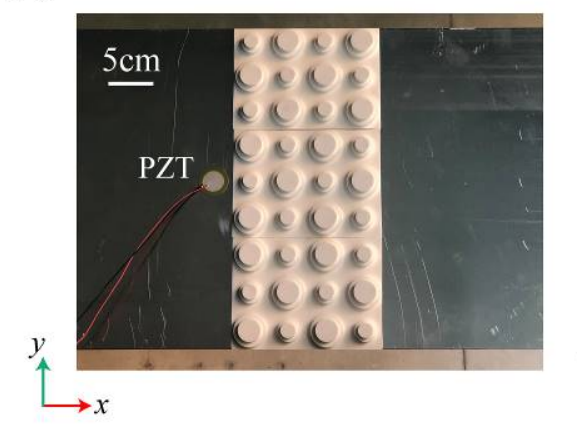

(c)

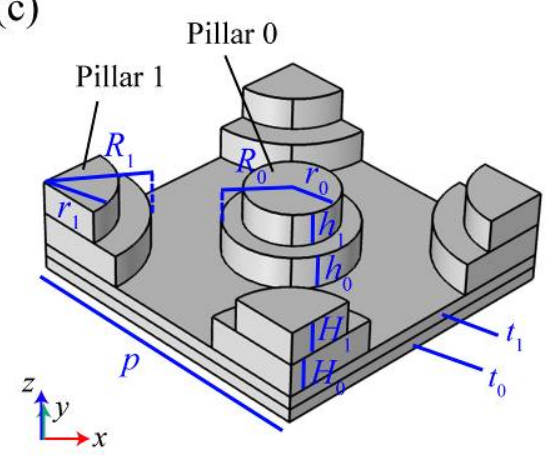

Fig. 1. Design of the bifunctional superlens. (a) Schematic diagram of the superlens for flexural and acoustic wave. A phononic crystal is glued on the aluminum thin plate. The unit cell of the phononic crystal is marked by red region. (b) Photograph of the experimental setup. The phononic crystal is $3 \mathrm{D}$-printed by the material of PLA with a size of $12.5 \mathrm{~cm} \times 30 \mathrm{~cm} \times 1.2 \mathrm{~cm}$. The thin plate is made by aluminum with a size of $50 \mathrm{~cm} \times 30 \mathrm{~cm} \times 0.2 \mathrm{~cm}$. The piezoelectric transducer (PZT) is shown in the figure. A scale bar is shown in the figure. (c) The unit cell of phononic crystal, with the radius $R_{0}, r_{0}$ and $R_{1}, r_{1}$ of Pillars 0 and 1 , the height $h_{0}, h_{1}$ and $H_{0}, H_{1}$ of Pillars 0 and 1 , the thickness $t_{0}$ and $t_{1}$ of the aluminum plate and the PLA substrate.

The first ingredient to realize the superlensing effect is to seek for negative dispersion bands in the band structure of the phononic crystal [5]. Using the unit cells exposed in Figs. 2(a-c), we numerically compute the band structures (red circles in Figs. 2(d-f)) by COMSOL Multiphysics $5.4 \mathrm{a}$ of the elastic waves propagating in the plate attached to the phononic crystals, the acoustic 
wave propagating in the air surrounding the pillar structure, and for both elastic waves and acoustic wave propagating in the composite structure. The waves travel through the phononic crystal along the $x$-direction which corresponds to the $\Gamma \mathrm{M}$ path of the first Brillouin zone. In Fig. 2(d), one can see the existence of a branch for elastic waves with negative slope within 4.8$9.1 \mathrm{kHz}$ range. The $\Gamma$ and $\mathrm{M}$ points in this mode $(k=0.02$ and $k=1)$ are marked in the figure by blue circles. It is noted that for the range of $k=0.02-0.38$ (viz., 7.4-9.1kHz), the curve is with positive slope, while for the range of $k=0.4-1$ (viz., $4.8-9.1 \mathrm{kHz}$ ), the curve is with negative slope. Thus, the frequency range of $4.8-7.4 \mathrm{kHz}$ marked by blue shadow is a pure negative refraction region for this mode. The dispersion curve of the flexural wave propagating in the aluminum plate is plotted (black line) in Fig. 2(d). The crossing point between the negative refraction curve and the dispersion curve of aluminum plate (near point A) indicates that the flexural wave propagating in the aluminum plate will be coupled to the phononic crystal array and be converted to a high-order elastic phononic mode with negative refraction phenomenon. The dispersion curve of aluminum plate at the crossing point has been folded several times in the band structure of phononic crystal. This can be numerically explained by the fact that the designed lattice constant $(p=4.75 \mathrm{~cm})$ of the phononic crystal part is larger than the wavelength of acoustic and flexural waves.

Similarly, In Fig. 2(e), the dispersion curve of the sound wave propagating in the air is plotted (blue line) in Fig. 2(d). A branch for acoustic waves with negative slope within 6.9$9.0 \mathrm{kHz}$ range is marked by blue shadow. Combining the band structures for elastic and acoustic waves in Figs. 2(d-e) implies that the joint negative refraction band is within the range of 6.97.4kHz. The working frequencies are selected in this range, and marked by points $\mathrm{A}(7.1 \mathrm{kHz})$ and $\mathrm{B}(7.25 \mathrm{kHz})$ for elastic wave and acoustic wave, respectively. The band structure for the 
composite structure for both elastic and acoustic waves are shown in Fig. 2(f). In this figure, the unbounded acoustic mode is marked by the yellow color. Such an unbounded acoustic mode is located in the frequency range $(>6.4 \mathrm{kHz})$ where the dispersion curve of the acoustic wave has higher $k$ value than that of flexural wave. The chosen working points A and $\mathrm{B}$ are located in the unbounded zone, indicating that the elastic mode used in this study is within the acoustic cone and thus would radiate acoustic energy from plate to air. This allows the exchange between elastic energy and acoustic energy, and makes some coupled elasto-acoustic mode possible. The simulations of the negative refraction in the phononic crystal for flexural and acoustic wave with plane wave incidences are shown in Supplementary Note 1.

(a)

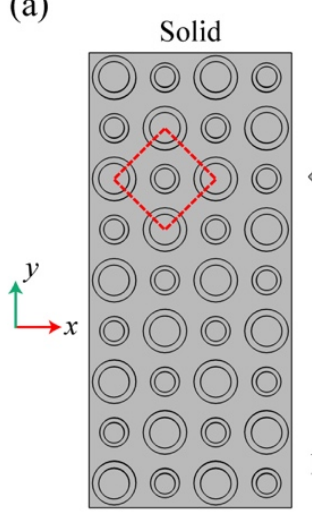

(d)

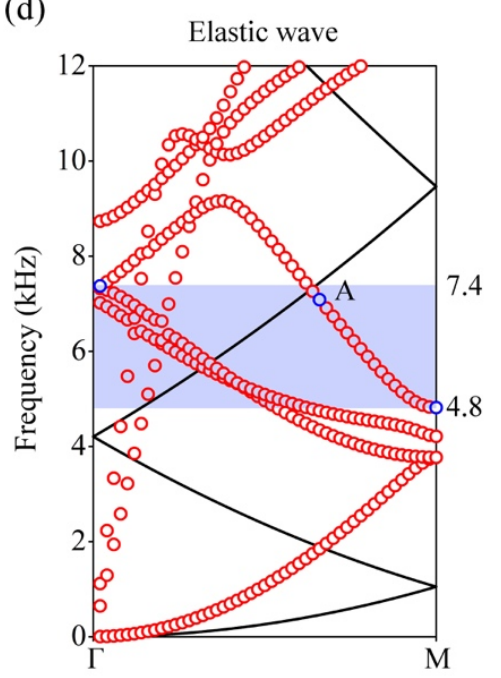

(b)

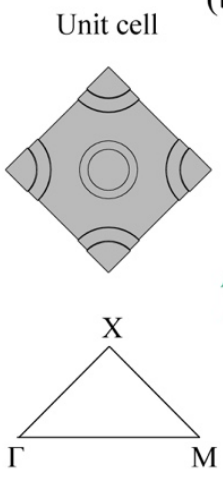

(e)
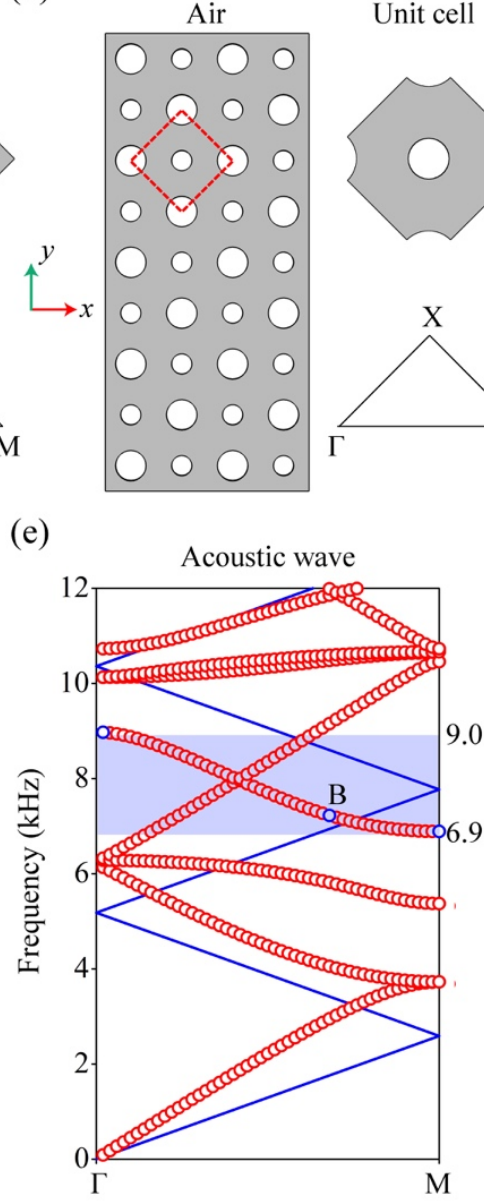

Unit cell (c)

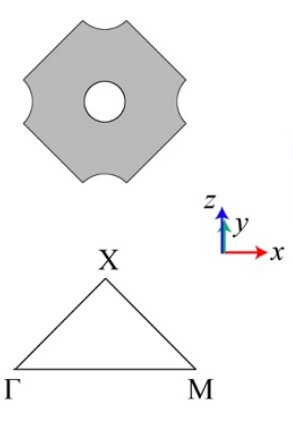

Composite structure

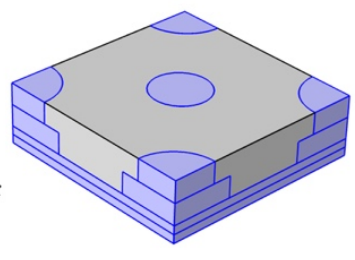

(f)

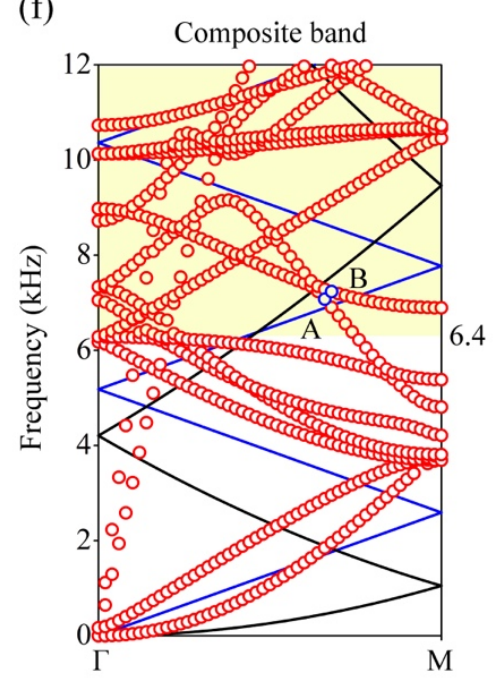


Fig. 2. The band structures. The schematic diagram of the phononic crystal for (a) flexural wave in the soild (b) acoustic wave in the air (c) two waves in composite structure. The unit cell of the square lattice is marked by red dashed line. (d-f) The band structures of the square lattice for flexural waves and acoustic waves are plotted by red circles. The dispersion curves of the flexural wave in the aluminum plate and the airborne wave without phononic crystal are plotted with black lines. The negative refraction bands are marked by blue shadows. The unbounded zone $(>6.4 \mathrm{kHz})$ is marked by yellow color, indicating that the plate could radiate acoustic energy into air.

The second ingredient to realize the superlensing effect is to seek for edge modes in the supercell to ensure the enhancement of evanescent waves at the interface between the finite phononic crystal and the surrounding medium. Figures 3(a-c) show the simulated eigenmodes of the supercells in the solid, air, and composite structure, respectively. In these numerical simulations, the periodic conditions are applied on the upper and bottom boundaries, as marked by the blue lines. Red arrows indicate the propagating direction ( $x$-direction). Black arrows indicate the enhancement of evanescent waves.

For the simulations of the single wave in Figs. 3(a-b), the $z$-component displacement distribution and acoustic pressure distribution are displayed. The edge resonant modes are observed at the frequency of $7.1 \mathrm{kHz}$ and $7.25 \mathrm{kHz}$ for elastic and acoustic waves, indicating that the evanescent waves can be enhanced at the interface of the array marked by the black arrows. The two working frequencies are within the joint negative band of $6.9-7.4 \mathrm{kHz}$, and are located at the frequency of A and B $(7.2 \mathrm{kHz})$ in Figs. 2(d-f). This means that we have a good coupling between the incident wave, the negative refraction wave in the phononic crystal, and the edge 
modes that will enhance evanescent wave. This agreement is achieved by choosing the optimized structural parameters of the Pillars 0 and 1.

Figure 3(c) shows the simulations of simultaneous elastic and acoustic waves in the composite structure. We can see that for the mode at $7.1 \mathrm{kHz}$ (corresponding to mode A in Fig. 2), edge modes can be observed in both elastic domain and acoustic domain. However, at $7.25 \mathrm{kHz}$, (corresponding to mode $\mathrm{B}$ in Fig. 2) edge modes can only be observed in acoustic domain, but disappears in elastic domain. We can conclude that mode A at $7.1 \mathrm{kHz}$ is the coupled mode that supports edge modes for both elastic and acoustic waves, but mode B at $7.25 \mathrm{kHz}$ is not coupled and only supports edge mode for acoustic wave. The field distribution in the lattices of the eigenmodes at the frequency $7.1 \mathrm{kHz}$ and $7.25 \mathrm{kHz}$ for elastic and acoustic wave are shown in Figs. 3(d-e), corresponding to the points A and B in Figs. 2(c-d) with $k=0.66$ and $k=0.68$, respectively.

(a)
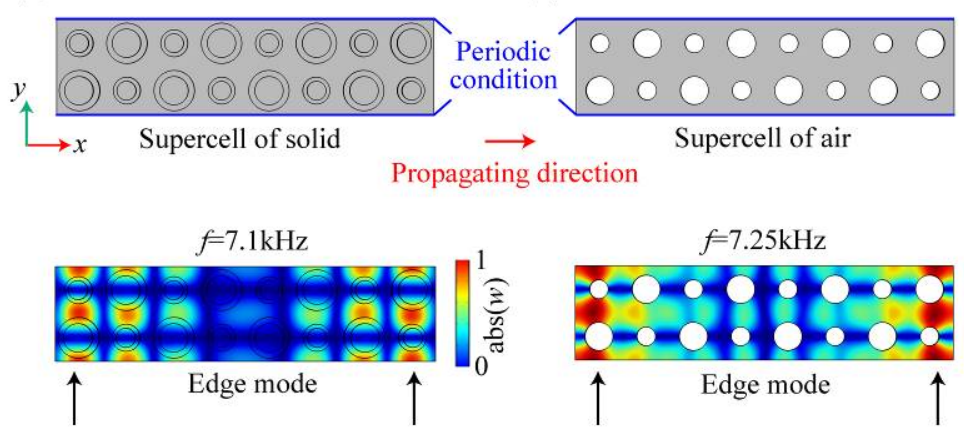

(d)

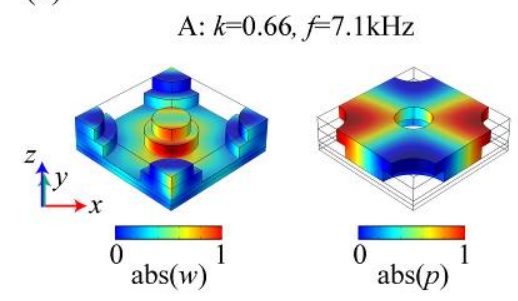

(b)

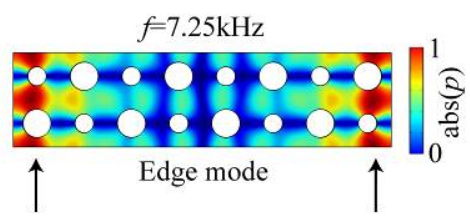

(e)

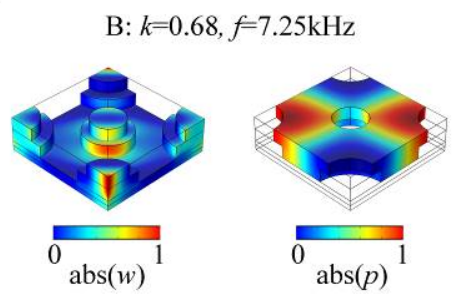

(c)

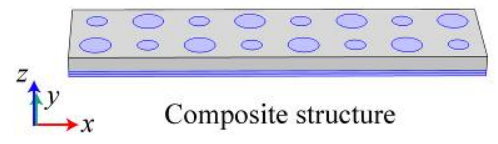

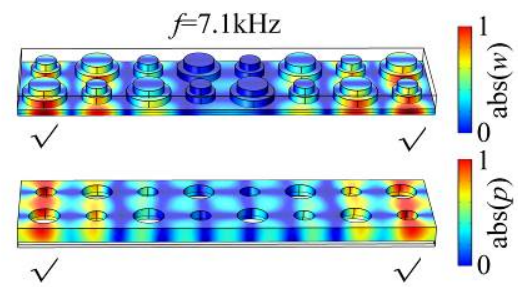

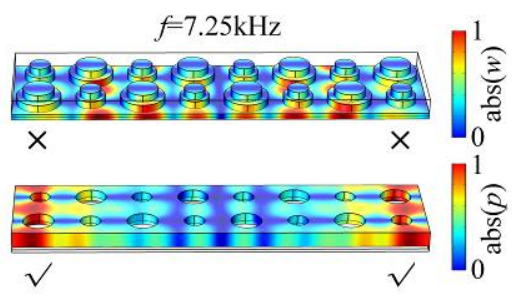

Fig. 3. Numerical simulation of the edge modes. (a) The eigenmode of the edge mode for the 
supercell at $7.1 \mathrm{kHz}$ for elastic wave. The color scale gives the $z$-component displacement distribution $[\operatorname{abs}(w)]$. (b) The eigenmode of the edge mode for the supercell at $7.25 \mathrm{kHz}$ for acoustic wave. The color scale gives the acoustic pressure amplitude distribution $[\operatorname{abs}(p)]$. Periodic conditions are applied on the top and bottom boundaries in simulation as marked by blue lines. Red arrows indicate the propagating direction ( $x$-direction). Black arrows indicate the enhancement of evanescent waves. (c) The eigenmode for the composite structure at $7.1 \mathrm{kHz}$ and $7.25 \mathrm{kHz}$, respectively. The $z$-component displacement distribution in the solid and acoustic pressure amplitude distribution in the air and are shown. The edge mode can be observed for simultaneous elastic wave and acoustic wave at $7.1 \mathrm{kHz}$, but can be observed only for acoustic wave at $7.25 \mathrm{~Hz}$. (d-e) The bulk eigenmode for elastic wave at $7.1 \mathrm{kHz}$ and acoustic wave at $7.25 \mathrm{kHz}$, corresponding to point A with $k=0.66$ and point B with $k=0.68$, respectively.

The physical conditions to produce superlensing effect for both elastic and acoustic waves are now established by designing the suitable phononic structure, verified by the realization of both negative refraction and the enhancement of the evanescent waves responsible to carry the sub-wavelength features. We then will show the numerical and experimental demonstration of our bifunctional superlens. Since the eigenfrequencies of edge mode for elastic and acoustic waves are adjacent $7.1 \mathrm{kHz}$ and $7.25 \mathrm{kHz}$, we choose $7.2 \mathrm{kHz}$ as the joint working frequency for the bifunctional superlens. Figure 4(a) shows the simulated and experimental intensity distributions (the square of $z$-component displacement, $w^{2}$ ) of the superlens for flexural waves at $7.2 \mathrm{kHz}$, evidencing a focusing spot. In the experiment, the source for flexural wave is a piezoelectric transducer (PZT) with a radius of $1.3 \mathrm{~cm}$ glued tightly on the plate as shown in Fig. 1(b), for achieving good couplings between the source and the plate. The PZT source is excited 
by signal generator and power amplifier with the output voltage of $20 \mathrm{~V}$. The flexural wave field measurements are accomplished by laser scanning with Polytec PSV-500 scanning head about $1.5 \mathrm{~m}$ away from the plate [not shown in Fig. 1(b)]. In the measurement, the size of the scanning region is $9 \mathrm{~cm} \times 3 \mathrm{~cm}(x=11.5-14.5 \mathrm{~cm}, y=-4.5-4.5 \mathrm{~cm})$. In the inset of Fig. $4(\mathrm{a})$, one can see the measured intensity field agrees well with the simulated one. Figure 4(b) exhibits the simulated and experimental normalized focal intensity along $y$-direction $(x=12.5 \mathrm{~cm}, y=-4.5-4.5 \mathrm{~cm})$. Horizontal error bars are provided, which is obtained from three sets of measured data. The error bars indicate the influence of the positional accuracy. The simulated spatial resolution of the superfocusing, defined as the full width at half maximum (FWHM), is $1.9 \mathrm{~cm}$ (viz., $0.36 \lambda_{1}$, $\lambda_{1}=5.21 \mathrm{~cm}$ is the wavelength of flexural wave in the aluminum plate at $7.2 \mathrm{kHz}$ ).. The realization of the superlensing emphasizes the role of the phononic crystal's edge modes in the amplification of the evanescent waves.

On the other hand, Fig. 4(c) presents the simulated and experimental acoustic intensity distributions of the superlens for the acoustic waves at $7.2 \mathrm{kHz}$. The simulation details for acoustic domain are shown in Supplementary Note 2. The PZT source for flexural wave could radiate sound energy into air due to the location in the unbounded region in Fig. 2(f), but the radiation efficiency is limited. We therefore choose the individual source for acoustic wave to ensure enough acoustic power. In the experiment, the source for acoustic waves is a small loudspeaker placed on the plate. The acoustic wave field measurements are accomplished by $1 / 8$ inch-diameter Brüel\&Kjær type-4138-A-015 microphone and Brüel\&Kjær PULSE Type 3160. The size of the analyzed region is $9 \mathrm{~cm} \times 4 \mathrm{~cm}(x=11-15 \mathrm{~cm}, y=-4.5-4.5 \mathrm{~cm})$. The measured acoustic field also agrees well with the simulated one, for which the subwavelength focusing spot is shown in Fig. 4(c). Figure 4(d) provides the simulated and experimental normalized focal 
intensity along $y$-direction $(x=12 \mathrm{~cm}, y=-4.5-4.5 \mathrm{~cm})$. The numerical FWHM is $2.1 \mathrm{~cm}\left(v i z .0 .44 \lambda_{2}\right.$, $\lambda_{2}=4.76 \mathrm{~cm}$ is the wavelength of acoustic wave at $7.2 \mathrm{kHz}$ ). This size is still subwavelength, and beyond the diffraction limit of $0.5 \lambda$, showing fine superlensing effect in the air. Furthermore, via the simulations, we find that the bifunctional superlens has a good performance in a certain bandwidth, within about 7.0 to $7.3 \mathrm{kHz}$. This bandwidth is within the joint negative refraction band $(6.9-7.4 \mathrm{kHz})$, also around the two eigenfrequencies of edge modes $(7.1 \mathrm{kHz}$ and $7.25 \mathrm{kHz})$.

In experiment, some factors can influence the performance of the superlens. For example, the material properties may be affected due to additively manufactured parts. In order to demonstrate the robustness of the proposed superlens, we provide numerical simulations with different parameters which consider the possible error in the 3D printing fabrication. In Figs. 4(ef), the elastic modulus of PLA and the size (radius) of the pillars, are varying from $90 \%$ to $110 \%$ of the original one [27-28]. The simulated FWHM for flexural wave in Figs. 4(e) is ranging from $0.36 \lambda_{1}$ to $0.4 \lambda_{1}$ with fluctuant parameters, and the simulated FWHM for acoustic wave in Figs. 4(f) is ranging from $0.44 \lambda_{1}$ to $0.46 \lambda_{1}$. The results suggest that the potential errors of the parameters lead to a very small influence on the superlensing effect, which ensure the good agreement between numerical simulation and experimental measurement. 
(a)

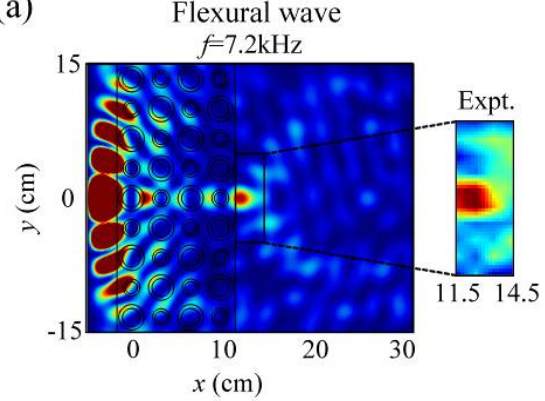

(b)

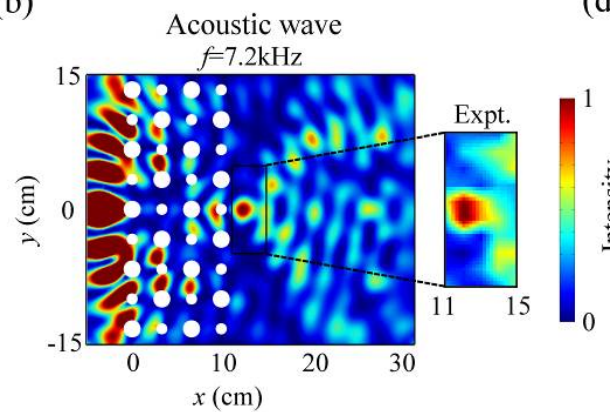

(c)

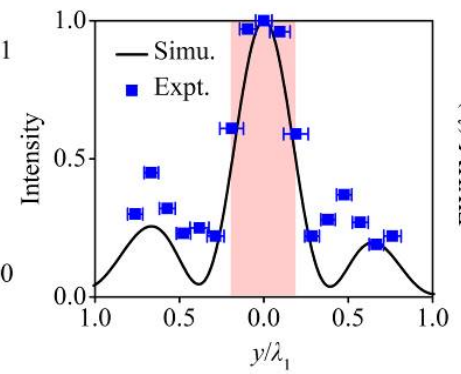

(d)

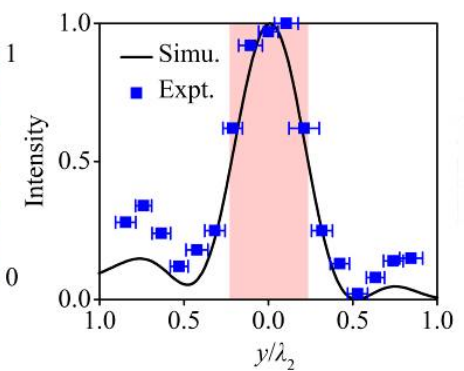

(e)

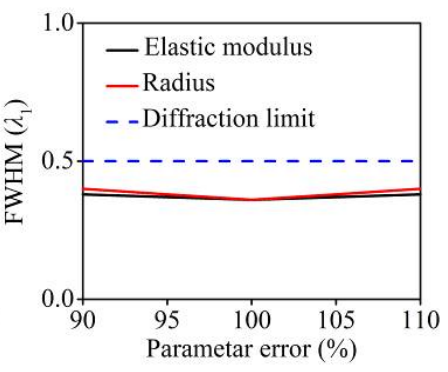

(f)

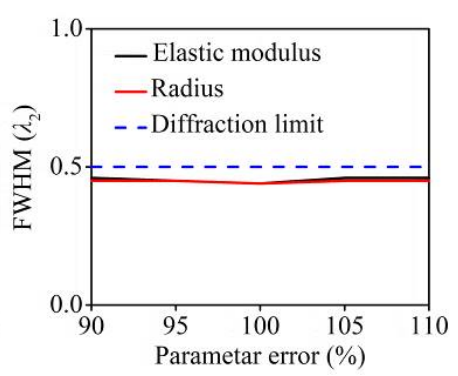

Fig. 4. Demonstration of bifunctional superlens. (a) The simulated and experimental acoustic intensity distributions of the superlens for flexural wave at $7.2 \mathrm{kHz}$. The size of the measuring region is $9 \mathrm{~cm} \times 3 \mathrm{~cm}(x=11.5-14.5 \mathrm{~cm}, y=-4.5-4.5 \mathrm{~cm})$ is indicated by the black rectangle. (b) The simulated (Simu.) and experimental (Expt.) normalized focal intensity along $y$-direction $(x=12.5 \mathrm{~cm}, y=-4.5-4.5 \mathrm{~cm})$. The numerical FWHM (red shadow) is $1.9 \mathrm{~cm}$, which corresponds to $0.36 \lambda_{1}$, where $\lambda_{1}=5.21 \mathrm{~cm}$ is the wavelength of flexural wave in the plate at $7.2 \mathrm{kHz}$. (c) The simulated and experimental acoustic intensity distributions of the superlens for acoustic wave at $7.2 \mathrm{kHz}$. The size of the measuring region is $9 \mathrm{~cm} \times 4 \mathrm{~cm}(x=11-15 \mathrm{~cm}, y=-4.5-4.5 \mathrm{~cm})$. (d) The simulated (Simu.) and experimental (Expt.) normalized focal intensity along $y$-direction $(x=12 \mathrm{~cm}, y=-4.5-4.5 \mathrm{~cm})$. The numerical FWHM (red shadow) is $2.1 \mathrm{~cm}$, which corresponds to $0.44 \lambda_{2}$, where $\lambda_{2}=4.76 \mathrm{~cm}$ is the wavelength of acoustic wave in the air at $7.2 \mathrm{kHz}$. Horizontal error bars are provided with three sets of measured data. (e) The simulated FWHM for flexural wave with fluctuant elastic modulus of PLA and fluctuant size (radius) of pillars. (f) The simulated FWHM for acoustic wave with fluctuant elastic modulus of PLA and fluctuant size 
(radius) of pillars.

To conclude, we have numerically and experimentally introduced a bifunctional superlens for simultaneous realization of flexural and acoustic wave superlensing by a single structure. The designed structure is a 3D-printed pillar-based phononic crystal array glued on a thin aluminum plate. Numerical studies have revealed that the designed phononic crystal can simultaneously produce negative refraction and edge modes to amplify the evanescent waves, which are the two necessary ingredients for superfocusing. By both numerical simulation and experimental measurements, the flexural and acoustic waves superfocusing are realized in the plate at $7.2 \mathrm{kHz}$. The FWHMs for flexural and acoustic waves focusing are $0.36 \lambda_{1}$ and $0.44 \lambda_{2}$, respectively. The proposed bifunctional superlens has a frequency bandwidth extending from $7.0 \mathrm{kHz}$ to $7.3 \mathrm{kHz}$. The multiwave focusing of the proposed phononic crystal has promissing applications in energy harvesting, since it can increase the total energy power at the interface between solid plate and the air medium. In addition, the proposed design strategy, viz., designing a structure that acts as rigid scatterers for the acoustic waves and as resonant scatterers for the flexural waves, could be extended to other bifunctional wave manipulations. Therefore, our findings may pave the wave to multiphysical and multifunctional devices [29-32].

See Supplemental Materials for the simulated negative refraction for flexural and acoustic waves, and the COMSOL simulation details.

\section{Acknowledgements}

This work is supported by the Air Force Office of Scientific Research under award number 
FA9550-18-1-7021, and by la Région Grand Est and Institut Carnot ICEEL.

\section{Data Availability Statement}

The data that support the findings of this study are available from the corresponding author upon reasonable request. 


\section{References}

[1] J. B. Pendry, Phys. Rev. Lett. 85, 3966-3969 (2000).

[2] M. Ambati, N. Fang, C. Sun, and X. Zhang, Phys. Rev. B 75, 195447 (2007).

[3] K. Deng, Y. Ding, Z. He, H. Zhao, J. Shi, and Z. Liu, J. Appl. Phys. 105, 124909 (2009).

[4] A. Sukhovich, B. Merheb, K. Muralidharan, J. O. Vasseur, Y. Pennec, P. A. Deymier, and J. H. Page, Phys. Rev. Lett. 102, 154301 (2009).

[5] X. Zhou, M. B. Assouar, and M. Oudich, Appl. Phys. Lett. 105, 233506 (2014).

[6] X. Zhou, M. B. Assouar, and M. Oudich, J. Appl. Phys. 116, 194501 (2014).

[7] J. Zhu, J. Christensen, J. Jung, L. Martin-Moreno, X. Yin, L. Fok, X. Zhang, and F. J. Garcia-Vidal, Nature Phys. 7, 52 (2011).

[8] N. Kaina, F. Lemoult, M. Fink, and G. Lerosey, Nature 525, 77 (2015).

[9] C. Ma, S. Kim and N. X. Fang, Nat. Commun. 10, 204 (2019).

[10] Y. X. Shen, Y. G. Peng, F. Cai, K. Huang, D. G. Zhao, C. W. Qiu, H. R. Zheng and X. F. Zhu, Nat. Commun. 10, 3411 (2019).

[11] J. J. Zhao, H. P. Ye, K. Huang, Z. N. Chen, B. W. Li, and C. W. Qiu, Sci. Rep. 4, 6257 (2014).

[12] X. Jiang, B. Liang, J. Yang, J. Yang, and J. C. Cheng, J. Appl. Phys. 123, 091717 (2018).

[13] M. A. Al-Lethawe, M. Addouche, A. Khelif, and S. Guenneau, New J. Phys. 14, 123030 (2012).

[14] T. Liu, F. Chen, S. J. Liang, H. Gao, and J. Zhu, Phys. Rev. Appl. 11, 034061 (2019).

[15] J. Xu, X. Jiang, N. Fang, E. Georget, R. Abdeddaim, J. Geffrin, M. Farhat, P. Sabouroux, S. Enoch, and S. Guenneau, Sci. Rep. 5, 10678 (2015).

[16] Y. Yang, H. Wang, F. Yu, Z. Xu, and H. Chen, Sci. Rep. 6, 20219 (2016). 
[17] C. Zhang, G. Y. Song, H. F. Ma, J. Yang, W. K. Cao, X. Y. Xie, P. Chen, Q. Cheng, L. T. Wu, T. J. Cui, Adv. Mater. Technol. 3, 1800161 (2018).

[18] Y. F. Zhu, X. Y. Zou, B. Liang, and J. C. Cheng, Appl. Phys. Lett. 107, 113501 (2015).

[19] S. W. Fan, S. D. Zhao, L. Y. Cao, Y. F. Zhu, A. L. Chen, Y. F. Wang, K. Donda, Y. S. Wang, and B. Assouar, Phys. Rev. B 101, 024104 (2019).

[20] A. Climente, D. Torrent, J. Sánchez-Dehesa, Appl. Phys. Lett. 105, 064101 (2014).

[21] Y. B. Jin, B. Bonello, R. P. Moiseyenko, Y. Pennec, O. Boyko, B. Djafari-Rouhani, Phys. Rev. B 96, 104311 (2017).

[22] L. Y. Cao, Y. L. Xu, B. Assouar, and Z. C. Yang, Appl. Phys. Lett. 113, 183506 (2018).

[23] L. Y. Cao, Z. C. Yang, Y. L. Xu, S. W. Fan, Y. F. Zhu, Z. L. Chen, B. Vincent, and B. Assouar Phys. Rev. Applied. 13, 014054 (2020).

[24] B. Assouar, B. Liang, Y. Wu, Y. Li., J. C. Cheng and Y. Jing, Nat. Rev. Mater. 3, 460-472 (2018).

[25] F. Legrand, B. Gérardin, J. Laurent, C. Prada, and A. Aubry, Phys. Rev. B 98, 214114 (2018).

[26] Q. Xie, S. Mezil, P. H. Otsuka, M. Tomoda, L. Jérôme, O. Matsuda, Z. Shen, and O. B. Wright, Nat. Commun. 10, 2228 (2019).

[27] S. Farah, D. G. Anderson, R. Langer Adv. Drug. Deliver. Rev. 107, 367-392 (2016).

[28] J. R. C. Dizon, A. H. Espera, Q. Y. Chen, R. C. Advincula, Addit. Manuf. 20, $44-67$ (2018).

[29] Y. F. Zhu and B. Assouar, Phys. Rev. B 99, 174109 (2019).

[30] Y. F. Zhu and B. Assouar, Phys. Rev. Mater. 3, 045201 (2019).

[31] Y. F. Zhu, F. Fei, S. W. Fan, L. Y. Cao, K. Donda and B. Assouar, Phys. Rev. Applied, 12, 034029 (2019). 
[32] H. Gao, X. S. Fang, Z. M. Gu, T. Liu, S. J. Liang, Y. Li, J. Zhu, Research, 1748537 (2019). 